\title{
Highly Sensitive Carbon Nanotube-Based Sensing for Lactate and Glucose Monitoring in Cell Culture
}

\author{
Cristina Boero*, Sandro Carrara, Giovanna Del Vecchio, Laura Calzà, and Giovanni De Micheli, Fellow, IEEE
}

\begin{abstract}
Monitoring of metabolic compounds in cell cultures can provide real-time information of cell line status. This is particularly important in those lines not fully known, as the case of embryonic and mesenchymal cells. On the other hand, such approach can pave the way to fully automated systems for growing cell cultures, when integrated in Petri dishes. To date, the main efforts emphasize the monitoring of few process variables, like $\mathrm{pH}, \mathrm{pO}_{2}$, electronic impedance, and temperature in bioreactors. Among different presented strategies to develop biosensors, carbon nanotubes exhibit great properties, particularly suitable for high-sensitive detection. In this work, nanostructured electrodes by using multiwalled carbon nanotubes are presented for the detection of lactate and glucose. Some results from simulations are illustrated in order to foresee the behavior of carbon nanotubes depending on their orientation, when they are randomly dispersed onto the electrode surface. A comparison between nonnanostructured and nanostructured electrodes is considered, showing that direct electron-transfer between the protein and the electrode is not possible without nanostructuration. Such developed biosensors are characterized in terms of sensitivity and detection limit, and are compared to previously published results. Lactate production is monitored in a cell culture by using the developed biosensor, and glucose detection is also performed to validate lactate behavior.
\end{abstract}

Index Terms-Carbon nanotubes, cell culture, electrochemical biosensors, electron transfer, metabolite monitoring.

\section{INTRODUCTION}

G LUCOSE AND lactate biosensors have been largely reported in literature for clinical purposes. A lot of solutions have been exploited to optimize the response of the sensor. For both the metabolites, electrodes have been structured with polymeric matrices, sol-gel, cross-linker, and mediators [1]. Recently, nanomaterials has been considered as possible electrical connectors to directly link the redox site to the electrode surface, because of the similar dimensions of nanoparticles and redox proteins. Various nanomaterials have been studied since the last ten years, including nanoparticles, nanowires and nanotubes [2].

Manuscript received September 03, 2010; accepted March 18, 2011. Date of current version April 27, 2011. This work was supported by the Swiss Science National Foundation under Grant IZLCZ2 123967 by Sino-Swiss cooperation) for the project "Nano-structured biochip development for stem cell monitoring". Asterisk indicates corresponding author.

${ }^{*} \mathrm{C}$. Boero is with the Integrated Systems Laboratory, École Polytechnique Fédérale de Lausanne, Switzerland (e-mail: cristina.boero@epfl.ch).

S. Carrara and G. De Micheli are with the Integrated Systems Laboratory, École Polytechnique Fédérale de Lausanne, Switzerland (e-mail: sandro.carrara@epfl.ch; giovanni.demicheli@epfl.ch).

G. Del Vecchio and L. Calzà are with the DIMORFIPA, Universitá di Bologna, Italy (e-mail: giovanna.delvecchio@yahoo.it; laura.calza@unibo.it).

Digital Object Identifier 10.1109/TNB.2011.2138157
Carbon nanotubes (CNTs) have aroused increasing interest for their electrical [3] and electrochemical properties [4] with special focus on biosensor applications [5]. It is possible to distinguish two large categories of CNTs: single-walled carbon nanotubes (SWCNT) are a rolled-up graphene single sheet with a diameter of typically about $2 \mathrm{~nm}$, which can behave either as a metal or a semiconductor; multiwalled carbon nanotubes (MWCNT) are graphene sheets rolled-up into concentric cylinders with external diameter in the range of $10-100 \mathrm{~nm}$. Due to the small diameter of these nanomaterials, quantum-effects dominate, and therefore these nanomaterials are efficiently coupled with proteins [6]. Studies have demonstrated that CNTs promote the electron-transfer between the enzyme redox active site and the transducing electrode [7].

Regarding oxidase-based biosensors, numerous studies were focused on the detection of metabolites like glucose, lactate, cholesterol, and glutamate. In the case of glucose the main application has been primarily related to diabetes pathology, in order to develop disposable devices for self-monitoring of blood glucose. Glucose detection was also interesting for other applications, like food industry for quality control purposes, or for keeping fermentation under control. Another metabolite of interest is lactate, which is related to anaerobic metabolism and it has been extensively studied in sport medicine, since it is correlated with muscle contraction, or pathological conditions which brings cell to suffer. In fact, elevated levels of lactate are mainly related to lack of feeding or hypoxia, and these levels can have toxic effects on cells. One of the purposes of the present study is to monitor lactate production over the time on a cell culture, and comparing it with the behavior of glucose uptake, to verify that lactate is secreted when there is a lack of feeding.

One possible strategy to develop biosensors for the aforementioned compounds is to employ amperometric enzyme-based biosensors. Oxidases belong to a large family of enzymes, which are able to transform such metabolites in other compounds with the production of hydrogen peroxide $\left(\mathrm{H}_{2} \mathrm{O}_{2}\right)$. $\mathrm{H}_{2} \mathrm{O}_{2}$ is an electrochemical specie oxidizable by means of a constant voltage. For glucose and lactate the enzymatic reaction is the following $(\mathrm{X}=\mathrm{L}$ or $\mathrm{G})$ :

$$
\begin{aligned}
X O D / F A D+\text { substrate } & \rightarrow X O D / F A D H_{2}+\text { product } \\
X O D / F A D H_{2}+O_{2} & \rightarrow H_{2} \mathrm{O}_{2}+X O D / F A D
\end{aligned}
$$

where gluconic acid and pyruvate are the enzymatic products for glucose and lactate, respectively, and FAD (flavin adenine dinucleotide) is the redox active site, where the exchange of electrons takes place. Since the stoichiometry of the reaction foresees one molecule of $\mathrm{H}_{2} \mathrm{O}_{2}$ for one molecule of substrate, it is possible to directly measure the concentration of hydrogen peroxide to obtain the concentration of glucose or lactate. Due 
to electrochemical properties of $\mathrm{H}_{2} \mathrm{O}_{2}$, the application of a potential of $+650 \mathrm{mV}$ causes the oxidation of hydrogen peroxide, according to the following reaction:

$$
2 \mathrm{H}_{2} \mathrm{O}_{2} \rightarrow 2 \mathrm{H}_{2} \mathrm{O}+\mathrm{O}_{2}^{+}+4 e^{-} .
$$

The four $e^{-}$released to the electrode generates a current, which intensity is proportional to $\mathrm{H}_{2} \mathrm{O}_{2}$ concentration. Both the reaction and the application of a potential can be carried out into an electrochemical cell. The cell consists typically of three electrodes connected with a potentiostat, which is able to record the current between the working electrode and the counter electrode, by fixing a potential between the working electrode and the reference electrode. The reaction of oxidation or reduction occurs onto the working electrode.

About the applications of such biosensors to cell cultures, to date the effort in this sense was concentrated to monitor process variables, like $\mathrm{pH}, \mathrm{pO}_{2}$, electronic impedance, and temperature in bioreactors [8]. Recently, Pemberton et al. [9] developed an amperometric biosensor to monitor glucose in a liver cell line. The authors report concentration values for different densities of seeded cells after $24 \mathrm{~h}$ of culture. Therefore, it is possible to envisage new emerging application areas for metabolite biosensors in cell monitoring. These biosensors can be instrumental in investigating unknown, or non-well-known cell lines, as embryonic or mesenchymal stem cells, also during proliferation or switching state, adding important information to the state-of-the-art of these cell families. Alternatively, such biosensors can be used to monitor cell cultures and to develop automated systems to manage cell cultures, saving time to human work.

The objective of the present work is the development of nanostructured electrodes by using MWCNT for the detection of lactate and glucose. Results from simulations regarding CNTs behavior are presented to demonstrate why this type of nanomaterials is suitable for biosensing. A comparison between nonnanostructured and nanostructured electrodes is investigated to demonstrate that detection is not possible without carbon nanotubes. Then, a fully characterization of the developed biosensors is reported, and performance of the proposed biosensors are compared with literature. Finally, nanostructured electrodes are functionalized with the enzyme probe and employed in a cell culture to monitor lactate production and glucose uptake. For this study the SN56 cell line is used, which derives from fusion of septal neurons of postnatal mice with murine neuroblastoma cells and it can be consider a suitable model to study the effects of hypoxia on brain cells.

\section{MAterials AND Methods}

\section{A. Chemicals}

Carbon paste screen-printed electrodes (SPE-model DRP-110) and multiwalled carbon nanotubes were purchased from Dropsens (Spain). The electrodes are made of a graphite working electrode, which presents an active area equal to 13 $\mathrm{mm}^{2}$, a counter electrode, also made of graphite, and a reference electrode, which is made of $\mathrm{Ag} / \mathrm{AgCl}$. The total area of the cell is $22 \mathrm{~mm}^{2}$. Multiwalled carbon nanotubes (diameter 10 $\mathrm{nm}$, length $1-2 \mu \mathrm{m})$ were purchased in powder (90\% purity), and subsequently diluted in chloroform to the concentration of $1 \mathrm{mg} \mathrm{ml}^{-1}$ [2]. Samples were then sonicated in order to obtain an homogeneous solution.

Glucose oxidase from Aspergillus Niger (GOD, EC 1.1.3.4, 129.9 units/mg solid), lactate oxidase from Pediococcus species (LOD, EC 1.13.12.4, $\geq 20$ units/mg solid), D-(+)-glucose, and lithium L-lactate were purchased from Sigma-Aldrich (Switzerland) in lyophilized powder. All the proteins were dissolved in phosphate buffer solution (PBS) $0.01 \mathrm{M}$ at $\mathrm{pH}$ 7.4, while glucose and lactate were dissolved in Milli-Q.

\section{B. Cell Cultures}

SN56 cell line (clone SN56.B5.G4), derived from the fusion of septal neurons of postnatal day 21 mice with N18TG2 murine neuroblastoma cells [10], was a generous gift from Prof. Wainer (Emory University, Atlanta, GA). Proliferating cells were maintained in Dulbecco's modified Eagle's medium (DMEM, from Sigma-Aldrich, Switzerland), supplemented with $10 \%$ fetal bovine serum (FBS, Gibco/BRL, Rockville, MD), $2 \mathrm{mM}$ l-glutamine (from Sigma-Aldrich), and $40 \mathrm{U} \mathrm{ml}^{-1}$ penicillin/streptomycin (from Gibco), in $25 \mathrm{~cm}^{2}$ culture flasks (Corning, New York, NY) in a $5 \% \mathrm{CO}_{2}$ atmosphere at $37^{\circ} \mathrm{C}$. Medium in the stock flasks was changed every $48 \mathrm{~h}$ and the cells were subcultured when they reached $80 \%-90 \%$ of confluence.

For experiments, cells were seeded at $5 \times 10^{3}, 25 \times 10^{3}$, and $203 \times 10^{3}$ cells cm$~^{-2}$, after four passages from thawing. Then, cells were cultured onto uncoated wells in proliferating conditions for $48 \mathrm{~h}$. The surnatant medium was collected from the flasks at different cell densities after $0,4,24$, and $48 \mathrm{~h}$ after seeding. Each sample was then diluted 1:10 in PBS for electrochemical measurements. Then, samples were frozen, and thawed when measurements were performed.

\section{Preparation of Electrodes}

Nanostructured SPEs were prepared by using MWCNT and the probe enzymes. To prepare the MWCNT modified SPE, 40 $\mu 1$ of the MWCNT-chloroform solution wad deposited by drop casting ( $5 \mu 1$ each time) onto the working electrode and it was allowed to dry. Then, $20 \mu \mathrm{l}$ of glucose or lactate oxidase (15 $\mathrm{mg} \mathrm{ml}^{-1}$ and $125 \mathrm{mg} \mathrm{ml}^{-1}$, respectively) were dropped onto the working electrode and stored overnight at $+4^{\circ} \mathrm{C}$, in order to allow the adsorption of the proteins onto the electrode surface. Then, the drop was rinsed out with Milli-Q and the electrode was conditioned for $10 \mathrm{~min}$ at constant potential $(+550 \mathrm{mV})$ before the first use. All the functionalized electrodes were stored at $+4^{\circ} \mathrm{C}$ and covered with PBS, when not used.

\section{Apparatus}

The electrochemical response of electrodes is investigated by chronoamperometries under aerobic conditions. Electrochemical measurements were acquired by using Versastat 3 potentiostat (Princeton Applied Technologies). For calibration and investigation of the detection limit, the electrode was dipped into the PBS solution with a volume of $25 \mathrm{ml}$ under stirring conditions. A volume of $25 \mu \mathrm{l}$ per step of the target molecule was successively added into the solution with a time-step of $2 \mathrm{~min}$. In 


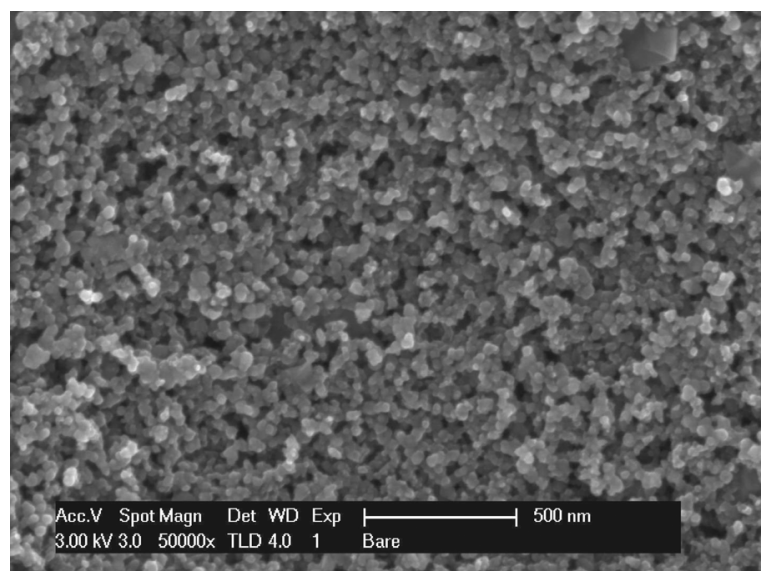

Fig. 1. SEM image of the surface of a bare electrode.

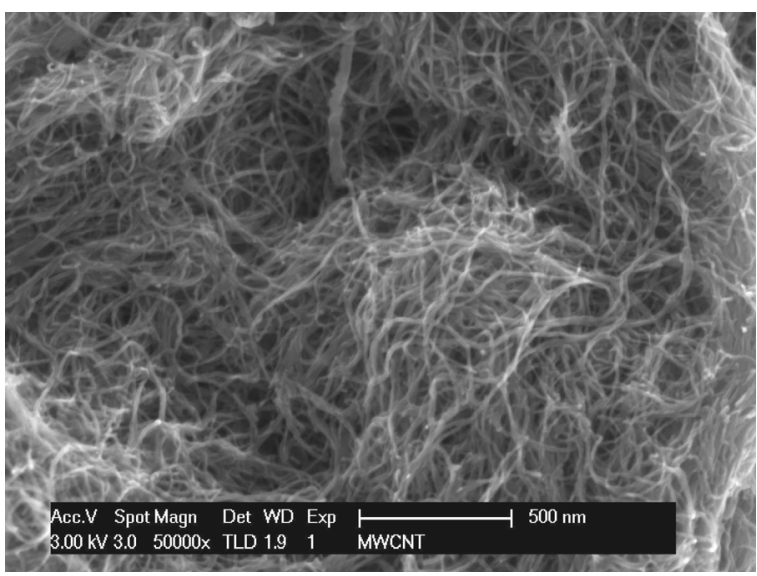

Fig. 2. SEM image of the surface of a nanostructured electrode.

the case of measurements of DMEM, the electrodes were covered with a drop of $100 \mu \mathrm{l}$ of the diluted medium. In both the cases, the applied potential was $+550 \mathrm{mV}$ vs $\mathrm{Ag} / \mathrm{AgCl}$.

A Philips/FEI XL-30 F microscope (Netherlands) was used to acquire scanning electron microscopic (SEM) images. The resolution in UHR mode is $2.5 \mathrm{~nm}$ at $1 \mathrm{kV}$.

\section{E. SEM Images}

Figs. 1 and 2 depict the acquired pictures for the bare electrode and for CNTs drop cast onto the electrode surface, respectively. As it is possible to see from the picture related to bare electrode, the surface has an important corrugation and graphite is characterized by small aggregates. On the other hand, when carbon nanotubes are dropped onto the surface, they form like "wrapped balls" of thin wires spread onto the surface. This behavior is due to the fact that the ends of carbon nanotubes, which terminate with a carboxylic group, are quite hydrophilic, but the walls, which comprise the majority of the tube, are highly hydrophobic. Therefore, they have the tendency to rapidly coagulate. Consequently, carbon nanotubes are dispersed in chloroform, that is a nonpolar organic solvent, but for the same reason, they also tend to be strongly adsorbed onto the electrode surface when chloroform evaporates [11].

\section{F. Theory of Carbon Nanotube Emissions}

It is already known that carbon nanotubes have considerable electron field emission properties. They are used to enhance the

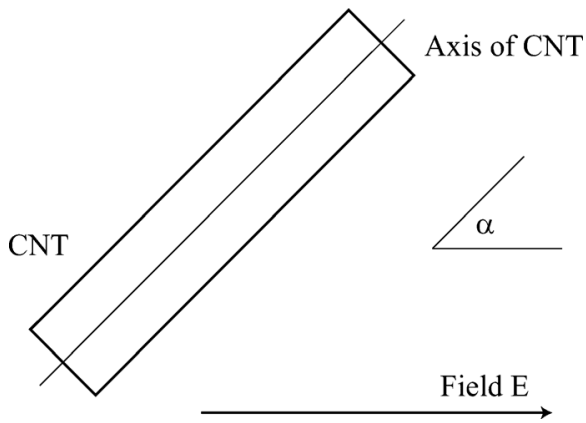

Fig. 3. Model for a linear segment of CNT.

emissivity of electrodes made of various materials [11]. CNTs are also used for enhancing sensitivity of biosensors [2], [11]. Many theoretical works have been published related to the field emission at the tip of individual capped CNTs, based both on the density functional theory or the Green's function theory [12], [13], or even using the symmetry properties of CNTs for solving the Schroedinger equation [14], [15]. On the other hand, experimental works have been made to investigate the sidewall field emission properties of CNTs aligned in bundle [16], [17]. Two conclusions were drawn in these works: the sidewall field emission obeys the Fowler-Nordheim equation [16], [17], and field emission performance increases with decreasing bundle diameter [17]. This property suggests that the best emission performance is from the body (the side wall) of individual CNTs. Our aim is to derive an equation, which governs the full emission of an individual CNT including electron emission from both sidewall and tip. The general equation needs to consider the arbitrarily oriented carbon nanotubes with respect to the field, while using a simple model to investigate nanostructured electrodes. Toward this goal, we investigate the situation in which there is a CNT with the axis parallel to the substrate and, therefore, perpendicular to field $E$. The developed model is based on the following three assumptions:

1) the electron emission occurs through the CNT half surface facing the anode (referring to Fig. 3);

2) the field $E$ is uniform both in direction and intensity in the neighborhood of the substrate and of the CNT;

3) the current emitted across the surface $\sigma$ obeys the Fowler-Nordheim equation considering the projection of $E$ on the normal to $\sigma$. In other words, assuming $\sigma$ as a flat surface, the Fowler-Nordheim equation is

$$
I=K_{1} \sigma E_{\perp}^{2} \exp \left(-\frac{K_{2}}{E_{\perp}}\right),
$$

where $I$ is the current emitted across $\sigma, E_{\perp}$ is the projection of $E$ on the normal to $\sigma$, and $K_{1}$ and $K_{2}$ are suitable constants. Then, if we consider an infinitesimal portion of the CNT surface, $d \sigma$, we have for the current

$$
d i=K_{1} d \sigma E_{\perp}^{2} \exp \left(-\frac{K_{2}}{E_{\perp}}\right) .
$$

Assuming a cylindrical coordinate system with the axis of $\mathrm{CNT}$ as z-axis, whose origin is at one end of CNT, and the direction of $E$ as origin of coordinate $\vartheta$, we have

$$
d \sigma=\rho d \vartheta d z ; \quad E_{\perp}=E \cos \vartheta,
$$


where $\rho$ is the radius of the carbon nanotube. By substituting (3) into (2), we obtain

$$
d i_{S}(E)=K_{1} \rho d \vartheta d z(E \cos \vartheta)^{2} \exp \left(-\frac{K_{2}}{E \cos \vartheta}\right) .
$$

The total current emitted across the side surface of the CNT is obtained by integrating on the portion of surface of CNT facing the anode

$$
\begin{aligned}
i_{S}(E) & =K_{1} \rho E^{2} \int_{0}^{L} d z \int_{-\frac{\pi}{2}}^{\frac{\pi}{2}} \cos ^{2} \vartheta \exp \left(-\frac{K_{2}}{E \cos \vartheta}\right) d \vartheta \\
& =K_{1} \rho E^{2} L \int_{-\frac{\pi}{2}}^{\frac{\pi}{2}} \cos ^{2} \vartheta \exp \left(-\frac{K_{2}}{E \cos \vartheta}\right) d \vartheta
\end{aligned}
$$

If the axis of CNT is not perpendicular to the field but forms an angle $\alpha$ (like in Fig. 3), (5) changes as follows:

$i_{S}(E, \alpha)=K_{1} \rho E^{2} L \int_{-\frac{\pi}{2}}^{\frac{\pi}{2}}(\cos \vartheta \sin \alpha)^{2} \exp \left(-\frac{K_{2}}{E \cos \vartheta \sin \alpha}\right) d \vartheta$.

In this case the emission from the CNT tip facing the anode should be considered. The current from the tip obeys the Fowler-Nordheim equation [14], which is written as

$$
i_{T}(E, \alpha)=K_{1}^{\prime} A(E \cos \alpha)^{2} \exp \left(-\frac{K_{2}^{\prime}}{E \cos \alpha}\right) .
$$

The entire current emitted by an oriented CNT forming an angle $\alpha$ with respect to the field is

$$
i(E, \alpha)=i_{S}(E, \alpha)+i_{T}(E, \alpha) .
$$

In the experimental activity on biosensors, possible parameter values may be $\rho=10 \mathrm{~nm}$ and $L=100 \mathrm{~nm}$, where $L$ is the linear shape of CNTs, confirmed by SEM image in Fig. 2, the applied field $E$ may range from $100 \mathrm{~V}$ to $1000 \mathrm{~V}$ and the emitted current from $0 \mu \mathrm{A}$ to about hundred of $\mu \mathrm{A}$. Therefore, suitable values for the constants that appear in Fowler-Nordheim equations were considered $K_{1}=10^{12}, K_{2}=35, K_{1}^{\prime}=(5 / 2) 10^{12}$ and $K_{2}^{\prime}=20$ by comparison with experiments performed in vacuum [16]. In case of a water environment, such as experiments with biosensors during the detection of glucose and lactate in the cell medium, the emitted current is further enhanced by the presence of water molecules close to nanotube surface [12].

\section{G. Monte Carlo Simulations}

In order to investigate the role of carbon nanotubes in our structured electrodes, numerical simulations are implemented considering (8). Specifically, Monte Carlo simulations are performed to obtain the final distribution of the carbon nanotubes onto our screen-printed electrodes. In fact, it is possible to imagine a situation such as the one shown in Fig. 4, where active redox species are in contact with all the available surface of a carbon nanotube drop cast onto the surface of a screen-printed electrode. In the figure, electron emission

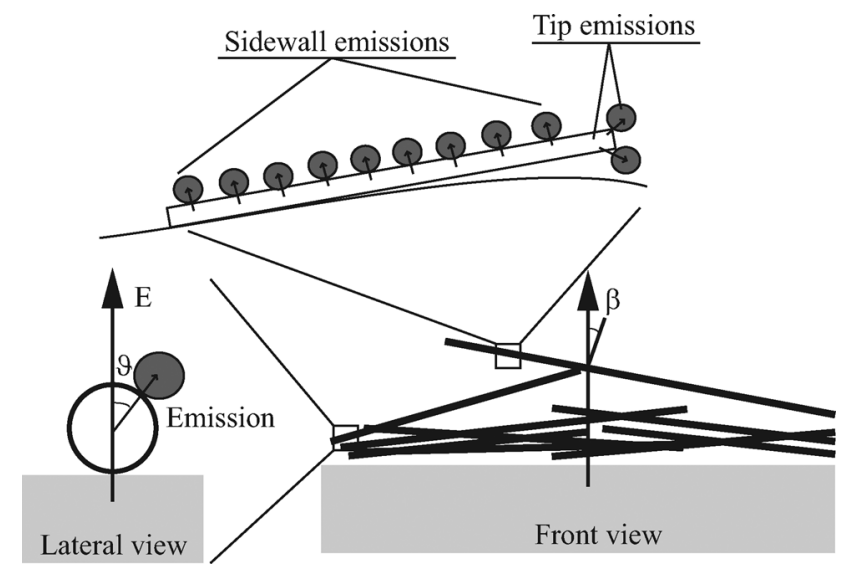

Fig. 4. Carbon nanotubes randomly organized onto the electrode surface and covered with proteins.

toward redox species would happen both from the sidewall and the tip. The electric field is typically normal to the baseline of the electrode. However, a screen-printed electrode has a mean corrugation in the 10-nm scale, as shown in Fig. 1. Then, the single carbon nanotube is positioned forming a certain angle with respect to the electric field, which would be typically normal to the electrode baseline, depending on the region where it was landing, as shown by Fig. 4. Therefore, Monte Carlo simulations are performed to simulate the landing of each single carbon nanotube onto the electrode surface. The final situation reached by each simulation is similar to the drawing in Fig. 4. The figure shows some carbon nanotubes differently organized onto the electrode surface, each of those presenting a different angle with respect to the electric field, which is orthogonal to the electrode surface.

\section{Results AND Discussion}

\section{A. Electron Emission From Carbon Nanotubes}

Initial results obtained from Monte Carlo simulations include the angle distribution of CNTs longitudinal axis with respect to the electrode surface. Fig. 5 illustrates such a distribution for one of the simulations. The large majority of carbon nanotubes is distributed to lower angles and the number of nanotubes with angles larger than $10^{\circ}$ decays very rapidly. Consequently, the sidewall emission decreases with the increasing angle of the nanotubes. Fig. 6 shows that the decay in the sidewall current is almost linear in a logarithmic scale, confirming first-order approximation of the sinus in (6), while the tip current follows a quadratic shape coherent with first approximation of the cosinus in (7). It is interesting to note that sidewall contribution to the total current is larger than tip contribution by orders of magnitude, even if current density is higher from the tip. Simulations shown in Fig. 6 allow to conclude that the tip emissions in drop cast CNTs are not negligible although the large majority of carbon nanotubes presents angles below $10^{\circ}$ with respect to the substrate.

\section{B. Improvements With Nanostructured Electrodes}

The improvements of nanostructured electrodes by using MWCNT were previously demonstrated in the case of hydrogen 


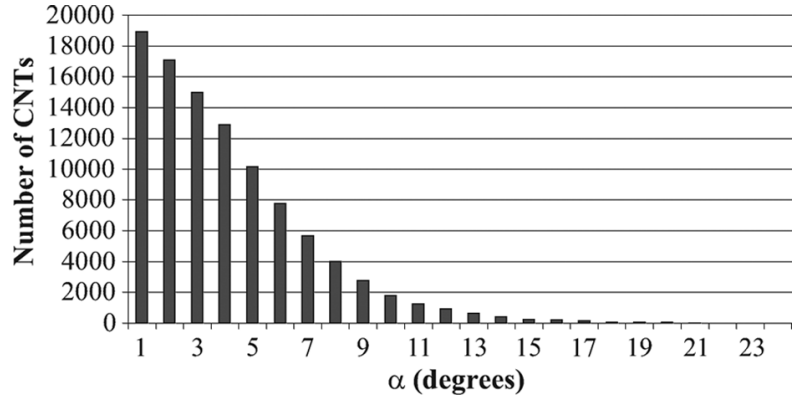

Fig. 5. Results from Monte Carlo simulation for the distribution of carbon nanotubes onto a flat surface.

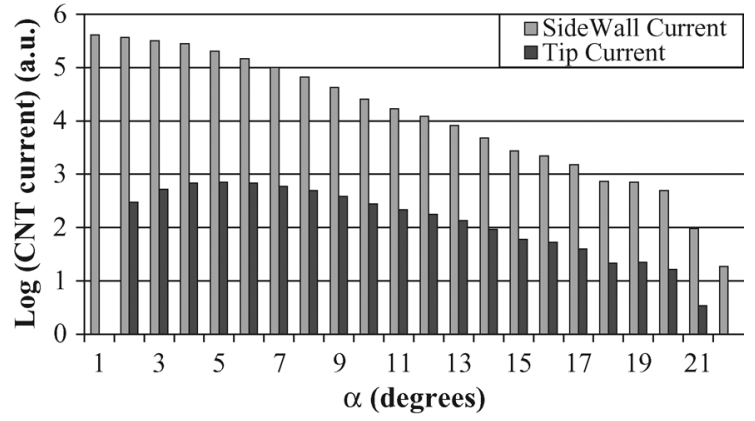

Fig. 6. Results from simulation regarding emission current from carbon nanotubes comparing the sidewall and the tip components, from (6) and (7).

peroxide [18]. The enhancement in terms of sensitivity between the case of nonnanostructured and nanostructured electrodes is about 7 times, also suggesting a similar improvement in terms of performance for oxidase-based biosensors.

Detection of lactate and glucose in the range of $\mathrm{mM}$ are performed for electrodes without and with nanostructuration, to evaluate the performance of the two strategies. The results are illustrated in Figs. 7 and 8. In the case of bare electrode, the detection is not possible within the range of interest. The detected current is in the order of $\mathrm{nA}$ for bare electrode, and signal to noise ratio is too high to sufficiently distinguish different concentrations of the substrate. On the other hand, the electrodes with carbon nanotubes show good sensitivity and a current range in the order of $\mu \mathrm{A}$. These results are coherent with literature [2]. Second-generation biosensors [1] overcame the difficulty to directly couple the enzyme with the electrode by using a mediator. The role of the mediator is shuttling electrons from the protein to the electrode surface, despite of the complexity of the system. From these results it is possible to assert that nanomaterials are good candidates to replace the role of mediators, since they allow the direct electron-transfer from the active site of the enzyme to the electrode surface. Moreover, chemical mediators are often bounded successively to the electrode surface, with problems related to mediator concentration and chemical bounding. On the contrary, nanomaterials can be grown onto the electrode surface during microfabrication, enabling integration with VLSI chips [19].

The range of detection is chosen considering that Hwang et al. [20] reported a mean value for lactate of $10.9 \mathrm{mM}$ in the case of murine embryonic stem cells. For the case of glucose, instead, the concentration in DMEM is at maximum $22.4 \mathrm{mM}$ (4.5 $\mathrm{g}^{-1}$ of glucose). Since it is not possible to perform measurements in pure DMEM, due to the interferences arising from

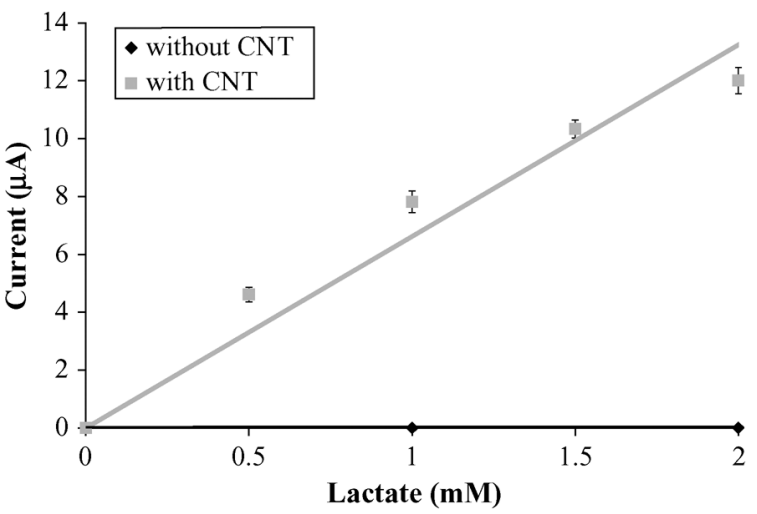

Fig. 7. Lactate detection for nonnanostructured and nanostructured electrodes.

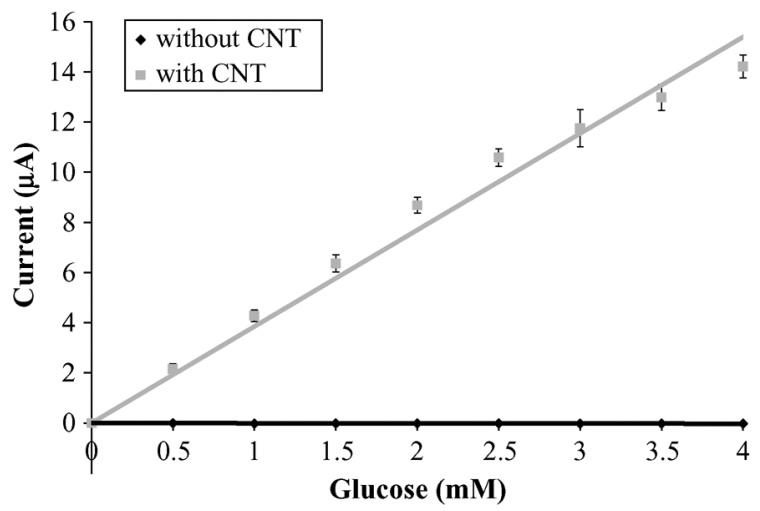

Fig. 8. Glucose detection for nonnanostructured and nanostructured electrodes.

easily electro-oxidizable substances at the same oxidizing potential, all the measurements are performed in diluted DMEM (dilution 1:10 in PBS), so that the maximum concentration of interest is $1 \mathrm{mM}$ for the case of lactate, and $2.24 \mathrm{mM}$ for the case of glucose. Moreover, linear range of the developed biosensor is not able to cover all the range of interest, so dilution is a proper solution. The range from 0.5 to $2.0 \mathrm{mM}$ for lactate and from 0.5 to $4.0 \mathrm{mM}$ for glucose are considered as the most suitable for the objectives of the present research, by considering the dilution of the DMEM.

\section{Calibration}

Calibration lines are worked out from chronoamperometries within the concentration range of interest (from 0.5 to $2.0 \mathrm{mM}$ for lactate and from 0.5 to $4.0 \mathrm{mM}$ for glucose, see Fig. 9). Both for lactate and glucose, substrates were dissolved in Milli-Q and added into the PBS solution every $120 \mathrm{~s}$, to allow the system to reach the steady-state. The response time of the system after each addition is around $30 \mathrm{~s}$ (data not shown). The calibration curve in the case of lactate detection shows a sensitivity of $40.1 \mu \mathrm{A} \mathrm{mM}^{-1} \mathrm{~cm}^{-2}$, while in the case of glucose detection the sensitivity is of $27.7 \mu \mathrm{A} \mathrm{mM}^{-1} \mathrm{~cm}^{-2}$. The detection limit for lactate biosensor is $28 \mu \mathrm{M}$, while it is $73 \mu \mathrm{M}$ for glucose, with a $\mathrm{S} / \mathrm{N}=3$ (see also Fig. 10). It is possible to compare the obtained sensitivities and detection limit with what was found in literature for similar nanostructured biosensors. Table I lists previous works regarding lactate detection. Note that the obtained sensitivity is twice the highest value reported in literature, as another indication that nanomaterials, such as carbon nanotubes, functionalized with oxidases are greatest substrate for biosensors. 


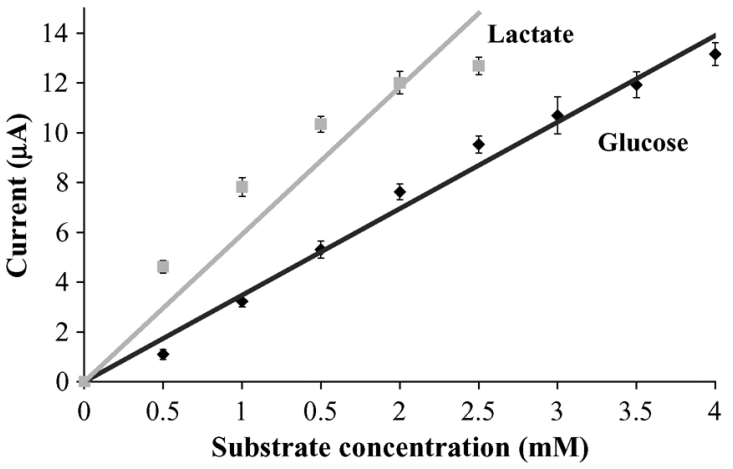

Fig. 9. Calibration lines for lactate and glucose in the concentration range of interest.

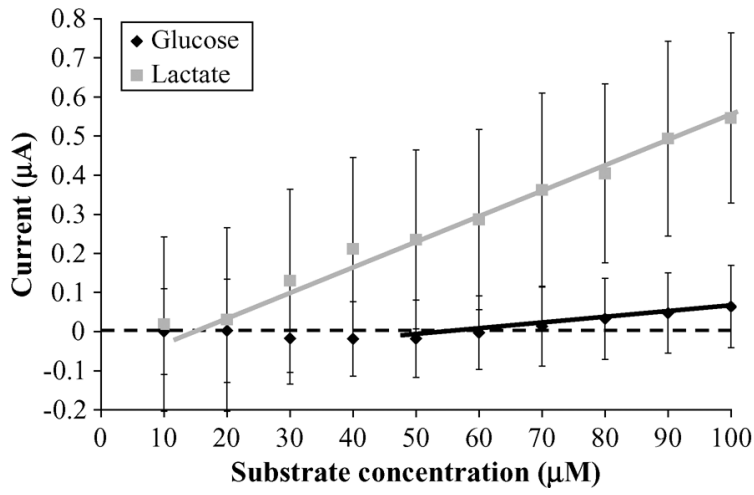

Fig. 10. Detection limit for lactate and glucose biosensors.

TABLE I

Performances of Nanostructured-Based Biosensors for Lactate DETECTION

\begin{tabular}{|c|c|c|}
\hline $\begin{array}{c}\text { Method for } \\
\text { lactate detection }\end{array}$ & $\begin{array}{c}\text { Sensitivity } \\
\left(\mu \mathrm{Am}^{-1} \mathrm{~cm}^{-2}\right)\end{array}$ & $\begin{array}{c}\text { Linear range } \\
(\mathrm{mM})\end{array}$ \\
\hline \hline LDH - MWCNT - CS [21] & 8.3 & $0.005-1.2$ \\
\hline MWCNT - PVI - CS [22] & 19.7 & $0.005-1$ \\
\hline MWCNT - sol-gel [23] & 2.097 & $0.3-2$ \\
\hline Present work & 40.1 & $0.028-2$ \\
\hline
\end{tabular}

TABLE II

Performances of NANOSTRUCTURED-Based Biosensors for Glucose DETECTION

\begin{tabular}{|c|c|c|}
\hline $\begin{array}{c}\text { Method for } \\
\text { glucose detection }\end{array}$ & 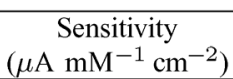 & $\begin{array}{l}\text { Linear range } \\
\text { (mM) }\end{array}$ \\
\hline MWCNT - Nafion(R) [24] & 171.2 & $0.005-0.5$ \\
\hline MWCNT - PtNP - CS/MTOS [25] & 69.9 & $0.012-6$ \\
\hline MWCNT - Nafion $\AA[26]$ & 4.7 & $0.025-2$ \\
\hline Present work & 27.7 & $0.073-4$ \\
\hline
\end{tabular}

Moreover, it is possible to assert that a further structuration of the electrode with polymers, as in the case of Cui et al. [22], does not exhibit better performance in terms of detection.

Regarding glucose detection, Table II shows a comparison among the sensitivities found in literature and in the present research. Rahman et al. [24] obtained the best sensitivity among the cited examples, but for a narrower detection range (from 1 to $500 \mu \mathrm{M}$ ), not useful for the purpose of the present work. Kang et al. [25] obtained a higher value of sensitivity, but they employed a sol-gel matrix combined with carbon nanotubes and nanoparticles, which can enhance the efficiency, despite of the cost and complexity of the sensor.

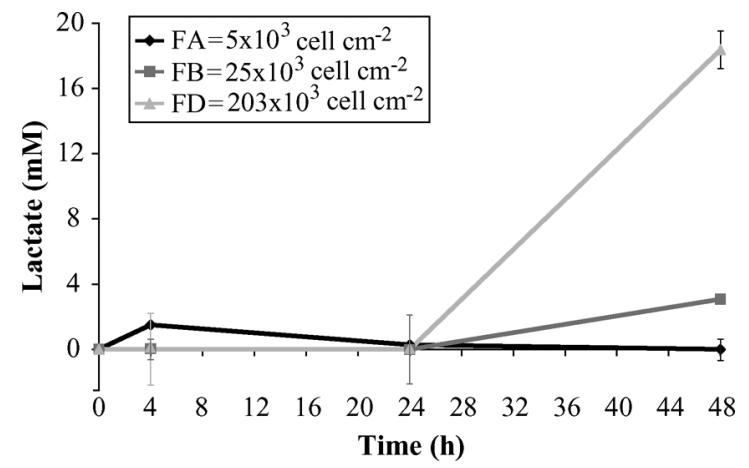

Fig. 11. Lactate production in cell line SN56 after 4, 24, and $48 \mathrm{~h}$.

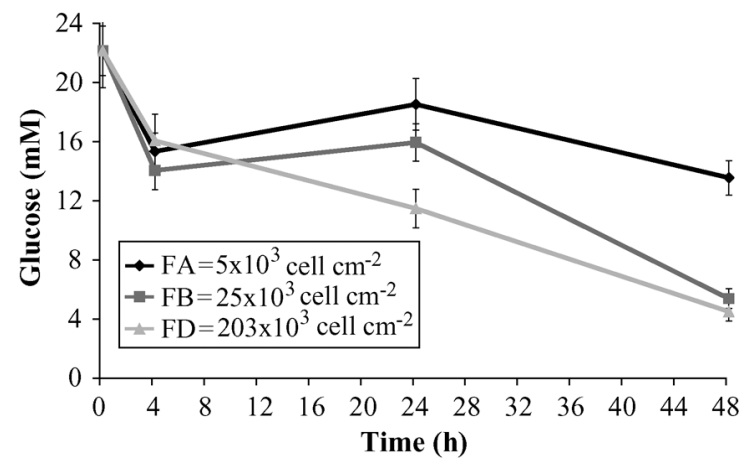

Fig. 12. Glucose uptake in cell line SN56 after 4, 24, and $48 \mathrm{~h}$.

These results also validate the phenomenon reported from Carrara et al. [6] regarding the inverse linear correlation between sensitivity and detection limit when using nanomaterials for biosensing. Comparing the obtained values in this work with nonnanostructured biosensors [27], [28], an improvement of performance with respect to the case of mediated electron-transfer is demonstrated. The advantages brought from nanomaterials are really evident, especially in ranges of concentration which are of interest for a large pool of applications, and they make carbon nanotubes the key-element for the future of nanobiosensing.

\section{Detection of Metabolites in Cell Cultures}

As mentioned above, DMEM is diluted with PBS in a rate of 1 to 10 , to be in the linear range of biosensors. Measurements are collected at the time of the seeding, and 4, 24, and $48 \mathrm{~h}$ later. The same diluted medium is used for lactate and glucose detection: the probe enzyme onto the electrode is different, but no cross talk or interferences are noticed from the other substrate during measurements. The behavior of lactate production and glucose uptake is depicted in Figs. 11 and 12 along $48 \mathrm{~h}$ of cell culture for different densities of cells.

Note that lactate production and glucose uptake are different for various cell densities. It means that with the developed biosensors is possible to recognize different cell densities and behaviors are clearly distinguishable. Another important point is that lactate production and glucose uptake shapes are quite symmetric. The lack of feeding, i.e., glucose, induces the production of lactate, which is a mark of cell suffering. Consequently, the symmetry of shapes is a further confirmation of this phenomena. On the other hand, in the lowest cell density the developed biosensor recorded an uptake of glucose in 
the first $4 \mathrm{~h}$, but afterwards the cells have enough feeding to survive more than $48 \mathrm{~h}$ without changing the medium. It is also confirmed in two ways by the fact that lactate production is almost zero. On the contrary, for the highest cell density, glucose uptake increases along time, while lactate production reached the maximum after $48 \mathrm{~h}$. Cells are still living, but the change of medium is required. From this perspective, the developed biosensors can be an useful instrument to investigate mechanisms occurring during cell proliferation, while simultaneously provide information to the operators about cell culture conditions and right timing for further cell feeding.

Moreover, observation of changes in the cell culture leads to develop skillful technologies for unknown cell systems. Biosensors developed in this study were tested and validated in a standard cell line, but such emerging devices can be applied to more complex cell lines, like stem cells, where there is a lack of knowledge about the mechanisms that unfold when cells switch from the stem state to the differentiate state.

It is necessary to improve the developed biosensors in order to make their results independent from system variables, like $\mathrm{pH}$, temperature, etc. For this reason, next work will be focused on enhancing accuracy of our sensors, in order to reduce variability among measurements.

\section{CONCLUSION}

This work presents the development of amperometric enzyme-based biosensors nanostructured by using multiwalled carbon nanotubes for metabolite detection in cell culture medium. In particular, biosensors are functionalized for the detection of lactate and glucose by means of oxidases. Results from simulations have demonstrated the role of carbon nanotubes in enhancing biosensing. Monte Carlo simulations are used to model the distribution of CNTs onto the electrode surface. Sidewall current and tip current are computed from the equation developed in the present research, based on the Fowler-Nordheim equation. Results show that there is an almost linear inverse relationship between the logarithm of the sidewall current and the angle formed by CNTs with the substrate, while the tip current follows a quasi-quadratic shape. From the point-of-view of simulation, tip emissions of randomly dispersed carbon nanotubes are not negligible even if the large majority of CNTs presents angles below $10^{\circ}$ with respect to the substrate.

Electrochemical measurements are performed for both metabolites by means of chronoamperometries. Sensitivity of $40.1 \mu \mathrm{A} \mathrm{mM}^{-1} \mathrm{~cm}^{-2}$ is reached in the case of lactate, while a value of $27.7 \mu \mathrm{A} \mathrm{mM}^{-1} \mathrm{~cm}^{-2}$ is obtained in the case of glucose detection. Detection limit is also investigated: a value of $28 \mu \mathrm{M}$ is obtained for lactate, while the detection limit for glucose is $73 \mu \mathrm{M}$. From a comparison with nanostructured biosensors by using carbon nanotubes in literature, the presented sensitivities are the highest value obtained among the previous researches in the case of lactate, while it is in the same order of magnitude of what was previously found in the case of glucose. These results are a further confirmation of how carbon nanotubes can improve the performance of biosensors. Once calibration is performed, detection of both these metabolites is performed in cell medium. Cells from SN56 line are seeded in three different densities and allow to proliferate for $48 \mathrm{~h}$. Then, a sample of the medium is collected at $0,4,24$, and $48 \mathrm{~h}$. Measurements are exploited with nanostructured electrodes functionalized with one of the two oxidases. Medium are diluted 1:10 in PBS to evaluate the concentration. Two behaviors are obtained, one for lactate production and one for glucose consumption. Lactate concentration increases along time, more in the case of higher cell density. Consequently, glucose level decreases in time, with a symmetric behavior, as assumed at the beginning. Such results illustrate the possibility to monitor metabolites in cell cultures by means of amperometric biosensors, enabling to develop fully integrated Petri dishes able to detect metabolites, targeting on automated system. On the other hand, the developed biosensors can add useful information to the knowledge of cell line, such embryonic or mesenchymal stem cells, which are not completely well-known.

Future work will focus on microfabrication of nanostructured electrodes and their functionalization with other oxidases, to detect other metabolite molecules.

\section{ACKNOWLEDGMENT}

The authors would like to thank Giuseppe D. Albini for his contribution to develop the model of electron emissions from carbon nanotubes and Monte Carlo simulations, and Dr. Vasileios Pavlidis for help in manuscript revision. The CIME facility is acknowledged for acquiring SEM images, especially Dr. Marco Cantoni and Ms. Fabienne Bobard.

\section{REFERENCES}

[1] R. Garjonyte, Y. Yigzaw, R. Meskys, A. Malinauskas, and L. Gorton, "Prussian blue- and lactate oxidase-based amperometric biosensor for lactic acid," Sens. Actuators B, vol. 79, no. 1, pp. 33-38, 2001.

[2] S. Carrara, V. Shumyantseva, A. Archakov, and B. Samorì, "Screenprinted electrodes based on carbon nanotubes and cytochrome p450 scc for highly sensitive cholesterol biosensors," Biosens. Bioelectron., vol. 24, no. 1, pp. 148-150, 2008.

[3] V. Bavastrello, E. Stura, S. Carrara, V. Erokhin, and C. Nicolini, "Poly(2, 5-dimethylaniline)-mwnts nanocomposite: A new material for conductometric acid vapours sensor," Sens. Actuators B, vol. 98, no. 2-3, pp. 247-253, 2004.

[4] V. Bavastrello, S. Carrara, M. Ram, and C. Nicolini, "Optical and electrochemical properties of poly(o-toluidine) multiwalled carbon nanotubes composite langmuirschaefer films," Langmuir, vol. 20, no. 3, pp. 969-973, 2004.

[5] S. Carrara, V. Bavastrello, D. Ricci, E. Stura, and C. Nicolini, "Improved nanocomposite materials for biosensor applications investigated by electrochemical impedance spectroscopy," Sens. Actuators $B$, vol. 109, no. 2, pp. 221-226, 2005.

[6] S. Carrara, C. Boero, and G. D. Micheli, "Quantum dots and wires to improve enzymes-based electrochemical bio-sensing," in Proc. Nanonet, 2009, vol. LNICTS 20, pp. 189-199, Berlin, Germany: Springer.

[7] C. Cai and J. Chen, "Direct electron transfer of glucose oxidase promoted by carbon nanotubes," Anal. Biochem., vol. 332, pp. 75-83, 2004.

[8] J. Wiest, M. Schmidhuber, J. Ressler, A. Scholz, M. Brischwein, and B. Wolf, "Cell based assays for diagnostic and therapy on multiparametric biosensor chips with an intelligent mobile lab," IFMBE Proc., vol. 10, pp. $132-135,2005$.

[9] R. Pemberton, J. Xu, R. Pittson, N. Biddle, G. Drago, S. Jackson, and J. Hart, "Application of screen-printed microband biosensors to endpoint measurements of glucose and cell numbers in hepg 2 cell culture," Anal. Biochem., vol. 385, no. 2, pp. 334-341, 2009.

[10] H. Lee, D. Hammond, T. Large, and B. Wainer, "Immortalized young adult neurons from the septal region: Generation and characterization," Dev. Brain Res., vol. 52, no. 1-2, pp. 219-228, 1990. 
[11] J. Gooding, "Nanostructuring electrodes with carbon nanotubes: A review on electrochemistry and applications for sensing," Electrochim. Acta, vol. 50, no. 15, pp. 3049-3060, 2005.

[12] L. Qiao, W. T. Zheng, Q. B. Wen, and Q. Jiang, "First-principles density-functional investigation of the effect of water on the field emission of carbon nanotubes," Nanotechnology, vol. 18, no. 15, p. 155707 , 2007, 6 pp.

[13] H. Roohi and S. Bagheri, "Atomic and electronic structures of finite single-walled bn nanotubes: Hybrid DFT calculations," J. Mol. Struct., vol. 856 , no. $1-3$, pp. 46-58, 2008

[14] A. Mayer, N. M. Miskovsky, and P. H. Cutler, "Theoretical comparison between field emission from single-wall and multi-wall carbon nanotubes," Phys. Rev. B, vol. 65, no. 15, p. 155420, 2002.

[15] A. Mayer and J.-P. Vigneron, "Real-space formulation of the quantummechanical elastic diffusion under $n$-fold axially symmetric forces," Phys. Rev. B, vol. 56, no. 19, pp. 12 599-12 607, 1997.

[16] Y. Chen, D. Shaw, and L. Guo, "Field emission of different oriented carbon nanotubes," Appl. Phys. Lett., vol. 76, no. 17, pp. 2469-2471, 2000.

[17] D. McClain, M. DeRoss, N. Tavan, J. Jiao, C. McCarter, R. Richards, S. Mesarovic, C. Richards, and D. Bahr, "Effect of diameter on electron field emission of carbon nanotube bundles," in Mater. Res. Soc. Symp. Proc., 2006, vol. 901E, pp. 251-256.

[18] C. Boero, S. Carrara, and G. De Micheli, "Sensitivity enhancement by carbon nanotubes: Applications to stem cell cultures monitoring," in Proc. Conf. Ph.D. Res. Microelectron. Electron. (PRIME), Jul. 2009, pp. $72-75$.

[19] A. Wisitsoraat, C. Karuwan, K. Wong-ek, D. Phokharatkul, P. Sritongkham, and A. Tuantranont, "High sensitivity electrochemical cholesterol sensor utilizing a vertically aligned carbon nanotube electrode with electropolymerized enzyme immobilization," Sensors, vol. 9, no. 11, pp. 8658-8668, 2009.

[20] Y. Hwang, J. Cho, F. Tay, J. Heng, R. Ho, S. Kazarian, D. Williams, A Boccaccini, J. Polak, and A. Mantalaris, "The use of murine embryonic stem cells, alginate encapsulation, and rotary microgravity bioreactor in bone tissue engineering," Biomaterials, vol. 30, no. 4, pp. 499-507, 2009.

[21] Y. Tsai, S. Chen, and H. Liaw, "Immobilization of lactate dehydrogenase within multiwalled carbon nanotube-chitosan nanocomposite for application to lactate biosensors," Sens. Actuators B, vol. 125, no. 2, pp. 474-481, 2007.

[22] X. Cui, C. M. Li, J. Zang, and S. Yu, "Highly sensitive lactate biosensor by engineering chitosan/pvi-os/cnt/lod network nanocomposite," Biosens. Bioelectron., vol. 22, no. 12, pp. 3288-3292, 2007.

[23] J. Huang, Z. Song, J. Li, Y. Yang, H. Shi, B. Wu, J. Anzai, T. Osa, and Q. Chen, "A highly-sensitive l-lactate biosensor based on sol-gel film combined with multi-walled carbon nanotubes (mwents) modified electrode," Mater. Sci. Eng. C, vol. 27, no. 1, pp. 29-34, 2007.

[24] M. Rahman, A. Umar, and K. Sawada, "Development of amperometric glucose biosensor based on glucose oxidase co-immobilized with multi-walled carbon nanotubes at low potential," Sens. Actuators $B$, vol. 137, no. 1, pp. 327-333, 2009.

[25] X. Kang, Z. Mai, X. Zou, P. Cai, and J. Mo, "Glucose biosensors based on platinum nanoparticles-deposited carbon nanotubes in sol-gel chitosan/silica hybrid," Talanta, vol. 74, no. 4, pp. 879-886, 2008.

[26] Y. Tsai, S. Li, and J. Chen, "Cast thin film biosensor design based on a nafion backbone, a multiwalled carbon nanotube conduit, and a glucose oxidase function," Langmuir, vol. 21, no. 8, pp. 3653-3658, Apr. 2005.

[27] Y. Himuro, M. Takai, and K. Ishihara, "Poly(vinylferrocene-co-2-hydroxyethyl methacrylate) mediator as immobilized enzyme membrane for the fabrication of amperometric glucose sensor," Sens. Actuators B, vol. 136, no. 1, pp. 122-127, 2009.

[28] R. Pemberton, R. Pittson, N. Biddle, and J. Hart, "Fabrication of microband glucose biosensors using a screen-printing water-based carbon ink and their application in serum analysis," Biosens. Bioelectron., vol. 24, no. 5, pp. 1246-1252, 2009, selected Papers from the Tenth World Congress on Biosensors Shangai, China, May 14-16, 2008.

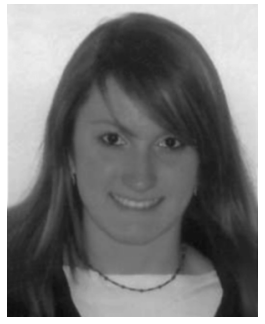

Cristina Boero received the M.Sc. degree in biomedical engineering from Politecnico di Torino, Italy, in 2007. She is currently working toward the Ph.D. degree in the Laboratory of Integrated Systems, École Polytechnique Fédérale de Lausanne (EPFL), Switzerland.

Her main research interest is in electrochemical biosensors for the detection of metabolites in stem cell cultures. The goal is to develop a fully integrate chip to detect such metabolites in real-time into Petri dishes. She is also interested in the functionalization of electrodes with nanostructures, like carbon nanotubes, and the interaction between proteins and nanostructures.

Ms. Boero received the Gold Leaf Award at PRIME in 2009.

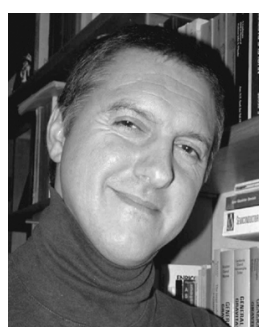

Sandro Carrara received the M.Sc. degree from University of Genoa, Italy, in 1993, and the Ph.D. degree in biochemistry and biophysics from University of Padoa, Italy, in 1997.

$\mathrm{He}$ is Senior Scientist and Lecturer at the École Polytechnique Fédérale de Lausanne (EPFL), Switzerland, and Professor of nanobiosensing and micro/nano interfaces at the Department of Electrical Engineering and Biophysics (DIBE), University of Genoa, Italy. He has more then 100 publications and 10 patents. He published Top 25 Hottest Articles (in 2004, 2005, 2008, and 2009) in highly ranked international journals. His main scientific interest is in electrical phenomena mediated by nanostructured molecular thin films and the development of protein and DNA based CMOS biochips.

Dr. Carrara is the founder and Editor-in-Chief of BioNanoScience and Associate Editor of the IEEE TRANSACTIONS ON BIOMEDICAL CIRCUITS AND SYSTEMS and of the IEEE SENSORS JOURNAL.

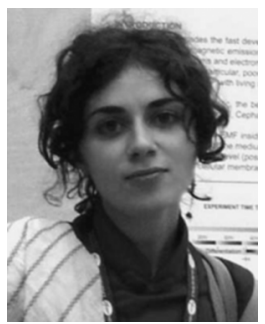

Giovanna Del Vecchio received the M.Sc. degree in medical biotechnologies and the $\mathrm{Ph} . \mathrm{D}$. degree in biotechnological tools for neuro-morpho-physiology from the University of Bologna, Italy, in 2004 and 2009, respectively.

From 2009 to 2010, she was a Postdoctoral Fellow at the University of Bologna and a Visiting Scientis at the EPFL, Switzerland. She is currently a Postdoctoral Fellow at the FMP-Leibniz Institute, Germany, working on the modulation of the blood brain barrier to improve the drug delivery in the CNS. She has coauthored 8 refereed journal papers and 19 conference papers and abstracts. Her research activity is mainly focused on CNS diseases through in vitro and in vivo models of neurodegeneration.

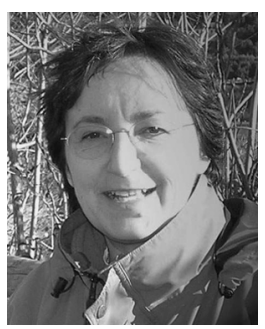

Laura Calzà received the Medical Science degree and the Specialist Training in endocrinology from the University of Modena, Italy, in 1980 and 1983, respectively.

She is Professor of Veterinary Anatomy, Director of the Interdipartimental Center for Industrial Research Health Science and Technology (ICIR-HST) at University of Bologna, Italy. She is a neurobiologist and an expert on in vitro and in vivo modeling for neurodegenerative diseases and dynamic imaging. She is author of more than 140 papers on referred journals. The main focus of her research activity is the study of reactive and repair mechanisms of the nervous system following experimental and spontaneous lesions, according to the bench-to-bedside approach of the translational medicine. Regenerative medicine for CNS using cell/scaffolds combined devices represents the most advanced area of interest. 


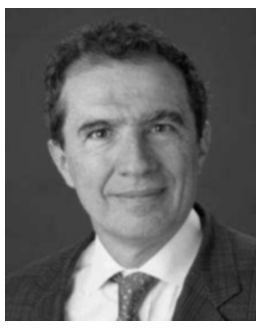

Giovanni De Micheli (F'94) received the nuclear engineer degree from Politecnico di Milano, Italy, in 1979, the M.Sc. and Ph.D. degree in electrical engineering and computer science from University of California, Berkeley, in 1983 and 1983, respectively.

$\mathrm{He}$ is Professor and Director of the Institute of Electrical Engineering and of the Integrated Systems Centre at EPFL, Lausanne, Switzerland. $\mathrm{He}$ is program leader of the Nano-Tera.ch program. His research interests include emerging technologies, networks on chips and 3-D integration. He is also interested in heterogeneous platform design including electrical components and biosensors, as well as in data processing of biomedical information.

Prof. De Micheli is a Fellow of ACM and a member of the Academia Europaea. He is the recipient of the 2003 IEEE Emanuel Piore Award, Golden Jubilee Medal from IEEE CAS Society in 2000, D. Pederson Award in 1987 for the best paper, two Best Paper Awards at the Design Automation Conference, in 1983 and in 1993, and a Best Paper Award at the DATE Conference in 2005. 